\title{
Error Analysis and Planning Accuracy for Dimensional Measurement in Active Vision Inspection
}

\author{
Christopher C. Yang, Member, IEEE, Michael M. Marefat, Senior Member, IEEE, \\ and Frank W. Ciarallo, Member, IEEE
}

\begin{abstract}
Spatial quantization error and displacement error are inherent in automated visual inspection systems. This paper discusses the effect of spatial quantization errors and displacement errors on the precision dimensional measurements for an edge segment. Probabilistic analysis in terms of the resolution of the image is developed for two-dimensional (2-D) quantization errors. Expressions for the mean and variance of these errors are developed. The probability density function (pdf) of the quantization error is derived. The position and orientation errors of the active head are assumed to be normally distributed. A probabilistic analysis in terms of these errors is developed for the displacement errors. Through integrating the spatial quantization errors and the displacement errors, we can compute the total error in the active vision inspection system. Based on the developed analysis, we investigate whether a given set of sensor setting parameters in an active system is suitable to obtain a desired accuracy for specific dimensional measurements. In addition, based on this approach, one can determine sensor positions and view directions which meet the necessary tolerance and accuracy of inspection.
\end{abstract}

Index Terms - Active vision, computer integrated inspection, dimensional inspection, displacement error, error analysis, image understanding, quantization error, scene analysis, threedimensional vision.

\section{INTRODUCTION}

\section{A. Background}

In machine vision inspection, the geometric features measured are those that are not changed with the environment or the set up of the vision system (the position and orientation of the camera). Examples of such invariant features are the length, width, area and volume of a pocket. Errors in measurement of invariant features by an automated, computer vision inspection system are inevitable. The sources of uncertainties that lead to these errors include:

1) displacement of the camera (position and direction);

2) quantization error in image digitization;

3) illumination error (poor contrast and low intensity);

4) motion of the object or the camera setup;

5) parallax (object to camera distance too small).

Manuscript received July 12, 1996; revised February 5, 1998. This paper was recommended for publication by Associate Editor K. M. Lee and Editor S. Salcudean upon evaluation of the reviewers' comments.

C. C. Yang is with the Department of Computer Science and Information Systems, University of Hong Kong, Hong Kong.

M. M. Marefat and F. W. Ciarallo are with the University of Arizona, Tucson, AZ 85721 USA.

Publisher Item Identifier S 1042-296X(98)03967-6.
The errors resulting from motion and parallax can be minimized to a negligible level by careful design and control of the environment. However, the errors due to the displacement of the sensor, quantization errors in image digitization, and illumination errors can not be avoided and always produce a significant effect on the measurement. Therefore, it is important that these errors be analyzed. The effect of these errors on the measurements can be minimized by carefully modeling the process leading to the errors.

\section{B. Review of Previous Work}

Previous research has introduced some results on spatial quantization errors. Kamgar-Parsi [15] developed the mathematical tools for computing the average error due to quantization, Blostein [2] analyzed the effect of image plane quantization on the three-dimensional (3-D) point position error obtained by triangulation from two quantized image planes in a stereo setup. Ho [11] expressed the digitizing error for various geometric features in terms of the dimensionless perimeter of the object, and Griffin [9] discussed an approach to integrate the errors inherent in the visual inspection.

In addition to quantization errors, in active vision inspection, the uncertainty arising from robot motion and sensory information is also important. Su et al. [30] presented a methodology for manipulating and propagating spatial uncertainties in a robotic assembly system for generating executable actions for accomplishing a desired task. Menq et al. [21] presented a framework to characterize the distribution of the position errors of robot manipulators over the work space and to study their statistical properties. Chen et al. [4] identified and parameterized the sources that contribute to the positioning error and estimated the values of the parameters. Veitschegger et al. [31] presented a calibration algorithm for finding the values of the independent kinematic errors by measuring the end-effector Cartesian positions and proposed two compensation algorithms: a differential error transform compensation algorithm and a Newton-Raphson compensation algorithm. Bryson [3] discussed methods for the measurement and characterization of the static distortion of the position data from 3-D trackers including least-squares polynomial fit calibration, linear lookup calibration, and bump lookup calibration. Smith et al. [29] presented a method for explicitly representing and manipulating the uncertainty associated with the transformation between coordinate frames representing the relative locations of objects. 
The previous research work has neither provided an analysis of the spatial quantization error for line segments in one and two dimensions nor has it investigated the effect of position and orientation errors in camera placement on the dimensional measurement of the object features. In addition, no analysis of expected accuracy in the dimensional measurements is provided.

Measurement accuracy involves the analysis of errors in the measurement of a manufactured product using a specific inspection strategy (for example, visual inspection, coordinate measurement machine, etc.). In this case, we analyze the accuracy using the measured value obtained in the inspection. For example, information from an image of a manufactured part is utilized in visual inspection. Planning accuracy involves the study of how the plan for inspection affects the accuracy of the inspection. In this case, we analyze the accuracy based on the inspection plan. The measured value of the product dimension(s) is not known because we only have the inspection plan without the execution of inspection. However, we do have the resolution of the camera and the planned sensor settings that can be used to inspect the dimensions for visual inspection. The error analysis of the dimensional measurement is based only on the probability density functions (pdf's) of the spatial quantization error of the image and the translational and orientational errors of the active vision head. This analysis gives us the capability to understand how to control the parameters of the sensor settings in order to increase the probability of high accuracy. Hence, in this paper, we study the planning accuracy in inspection.

\section{Contributions}

In this paper, we analyze the spatial quantization error in two dimensions. The pdf of the spatial quantization error of a point in one dimension has been modeled as a uniform distribution in previous work [9], [11]. Using a similar representation, we analyze the error in the measured dimension of a line in two dimensions. The mean, the variance, the range and the pdf of the error are derived. We also analyze the displacement error in an active vision system. Based on normally distributed translational and orientational errors of the active vision head [21], we analyze how these errors propagate to the displacement of the projected points from the 3 -D model to a two-dimensional (2-D) image plane. The pdf for the errors in dimensional measurement of linear segments resulting from displacement errors is derived. The total error in the active vision inspection is then characterized based on the integration of the quantization error and the displacement error. The expected accuracy in inspection of a set of entities by a given sensor setting is then analyzed. Using the predicted length, we can find the expected accuracy of the dimensional measurement in terms of the ideal dimension, the projected distance from the sensor to the line, and the angle of view.

\section{QUANTIZATION ERRORS IN INSPECTION}

The spatial quantization error is important in inspection, especially when the size of a pixel is significant compared to the allowable tolerances in the object dimension. A quantized

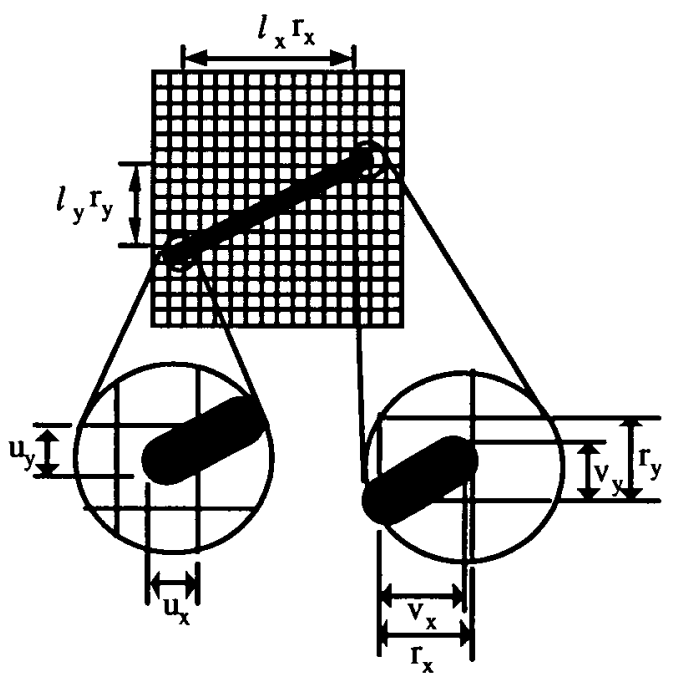

Fig. 1. A line on a 2-D array of pixels. The horizontal length of the line is $l_{x} r_{x}+u_{x}+v_{x}$. The vertical length of the line is $l_{y} r_{y}+u_{y}+v_{y}$.

sample indicates itself as part of the object image if and only if more than half of it is covered by the edge segment. Significant distortion can be produced by this kind of quantization. A point in the image can only be located to within one pixel of accuracy with traditional edge detection techniques. Recently, several new edge detection techniques have reported subpixel accuracy [12], [14], [16]-[18] to improve the precision of measurement on images. Many techniques in this group are based on interpolation of the acquired image. Although interpolationbased techniques increase the resolution of quantization to obtain sub-pixel accuracy, quantization still remains at some level.

\section{A. Two-Dimensional Quantization Errors in Dimensional Measurement}

The 2-D spatial quantization error is the combination of two one-dimensional (1-D) spatial quantization errors. Fig. 1 shows a line on a 2-D array of pixels. The resolution of the image is $r_{x} \times r_{y}$ where $r_{x}$ is the width of a pixel and $r_{y}$ is the length of a pixel. The horizontal component of the line length, $L_{x}$, is $l_{x} r_{x}+u_{x}+v_{x}$. The vertical component of the length of the line, $L_{y}$, is $l_{y} r_{y}+u_{y}+v_{y}$. The actual dimension, $L$, is therefore $\sqrt{L_{x}^{2}+L_{y}^{2}}$.

The quantized length, $L_{q}=\sqrt{L_{q_{x}}^{2}+L_{q_{y}}^{2}}$, where $L_{q_{x}}$ and $L_{q_{y}}$ are the horizontal and vertical quantized lengths, respectively. There are four random variables, two for the horizontal length, $u_{x}$ and $v_{x}$, and two for the vertical length, $u_{y}$ and $v_{y}$. All four are assumed to be uniformly distributed

$$
\begin{gathered}
f_{u_{x}}\left(u_{x}\right)=f_{v_{x}}\left(v_{x}\right)=\frac{1}{r_{x}} \quad \text { for } 0 \leq u_{x} \leq r_{x} \\
\text { or } 0 \leq v_{x} \leq r_{x} \\
f_{u_{y}}\left(u_{y}\right)=f_{v_{y}}\left(v_{y}\right)=\frac{1}{r_{y}} \quad \text { for } 0 \leq u_{y} \leq r_{y} \\
\text { or } 0 \leq v_{y} \leq r_{y} .
\end{gathered}
$$




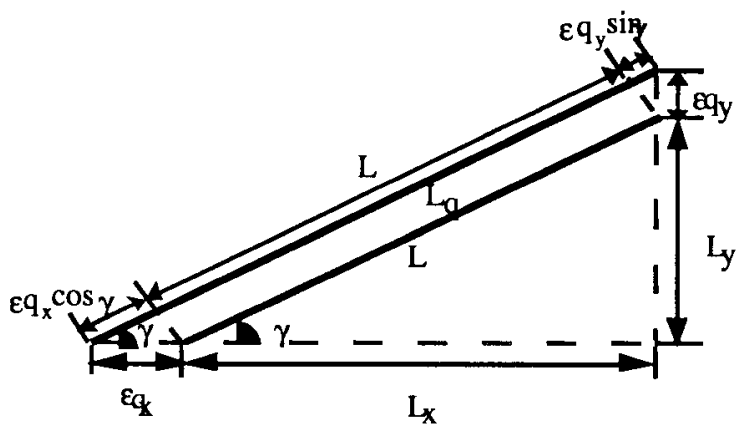

Fig. 2. Original line with length $L$ and angle between itself and horizontal axis $\gamma$ and the quantized line with length $L_{q}$ and parallel to the original line. (Figure is not shown in scale, $\varepsilon_{x}$ and $\varepsilon_{y}$ are much smaller than the length of the line in actual case.)

We will use a geometric approximation to characterize the 2-D quantization error. Fig. 2 shows a line with length $L$ and lying at an angle of $\gamma$ to the horizontal axis. (Note: The figure is not drawn to scale: $\varepsilon_{q_{x}}$ is much smaller than $L_{x}$ and $\varepsilon_{q_{y}}$ is much smaller than $L_{y}$.) If $\left(\varepsilon_{q_{x}}+L_{x}\right) /\left(\varepsilon_{q_{y}}+L_{y}\right)=L_{x} / L_{y}$, the quantized line is parallel to the original line. In this case, the length of the quantized line, $L_{q}$, is $L+\varepsilon_{q_{x}} \cos \gamma+\varepsilon_{q_{y}} \sin \gamma$ as shown in Fig. 2. The 2-D spatial quantized error, $\varepsilon_{q}$ (= $\left.L_{q}-L\right)$, is $\varepsilon_{q_{x}} \cos \gamma+\varepsilon_{q_{y}} \sin \gamma$ in this case.

However, if the original line and the quantized line are not parallel $\left(\left(\varepsilon_{q_{x}}+L_{x}\right) /\left(\varepsilon_{q_{y}}+L_{y}\right) \neq L_{x} / L_{y}\right)$, and the error, $\varepsilon_{q}$ is $\sqrt{\left(L \cos \gamma+\varepsilon_{q_{x}}\right)^{2}+\left(L \sin \gamma+\varepsilon_{q_{y}}\right)^{2}}-L$. Although the lines may not be exactly parallel, they are approximately parallel because $\varepsilon_{x}$ and $\varepsilon_{y}$ are very small compared to the length of the line $L$. Therefore

$$
\varepsilon_{q} \cong \cos \gamma \varepsilon_{q_{x}}+\sin \gamma \varepsilon_{q_{y}} .
$$

Using this geometric approximation, we can compute the mean and the variance of the 2-D quantization error. The mean of the quantization error in two dimensions is

$$
\begin{aligned}
E\left[\varepsilon_{q}\right] & \cong E\left[\cos \gamma \varepsilon_{q_{x}}+\sin \gamma \varepsilon_{q_{y}}\right] \\
& =\cos \gamma E\left[\varepsilon_{q_{x}}\right]+\sin \gamma E\left[\varepsilon_{q_{y}}\right]=0
\end{aligned}
$$

because $E\left[\varepsilon_{q_{x}}\right]$ and $E\left[\varepsilon_{q_{y}}\right]$ are both zero [34]. The variance of the quantization error in two dimensions is

$\operatorname{Var}\left[\varepsilon_{q}\right] \cong \operatorname{Var}\left[\cos \gamma \varepsilon_{q_{x}}+\sin \gamma \varepsilon_{q_{y}}\right]=\frac{1}{6}\left(\cos ^{2} \gamma r_{x}^{2}+\sin ^{2} \gamma r_{y}^{2}\right)$

because the variances of $\varepsilon_{q_{x}}$ and $\varepsilon_{q_{y}}$ are $1 / 6 r_{x}^{2}$ and $1 / 6 r_{y}^{2}$, respectively.

\section{B. Probability Density Function of Quantization Error}

In this section, we derive the pdf of the 2-D quantization error based on the geometric approximation. The pdf's of the errors in the horizontal and vertical directions are the following triangle distributions.

For the $x$-direction

$$
f_{\varepsilon_{q_{x}}}\left(\varepsilon_{q_{x}}\right)= \begin{cases}\frac{1}{r_{x}^{2}} \varepsilon_{q_{x}}+\frac{1}{r_{x}}, & -r_{x} \leq \varepsilon_{q_{x}} \leq 0 \\ -\frac{1}{r_{x}^{2}} \varepsilon_{q_{x}}+\frac{1}{r_{x}}, & 0 \leq \varepsilon_{q_{x}} \leq r_{x} \\ 0, & \text { otherwise. }\end{cases}
$$

For the $y$-direction

$$
f_{\varepsilon_{q_{y}}}\left(\varepsilon_{q_{y}}\right)=\left\{\begin{array}{cl}
\frac{1}{r_{y}^{2}} \varepsilon_{q_{y}}+\frac{1}{r_{y}}, & -r_{y} \leq \varepsilon_{q_{y}} \leq 0 \\
-\frac{1}{r_{y}^{2}} \varepsilon_{q_{y}}+\frac{1}{r_{y}}, & 0 \leq \varepsilon_{q_{y}} \leq r_{y} \\
0, & \text { otherwise. }
\end{array}\right.
$$

The approximate 2-D spatial quantization error, $\tilde{\varepsilon}_{q}$, using (1) is in terms of two variables $\varepsilon_{q_{x}}$ and $\varepsilon_{q_{y}}$. The pdf is expressed in terms of $\tilde{\varepsilon}_{q}$ and the joint statistics of $\varepsilon_{q_{x}}$ and $\varepsilon_{q_{y}}$

$$
\tilde{f}_{\varepsilon_{q}}\left(\tilde{\varepsilon}_{q}\right)=\frac{1}{|\cos \gamma|} \int_{-\infty}^{\infty} f\left(\frac{\tilde{\varepsilon}_{q}-\sin \gamma \varepsilon_{q_{y}}}{\cos \gamma}, \varepsilon_{q_{y}}\right) d \varepsilon_{q_{y}} .
$$

If $\varepsilon_{q_{x}}$ and $\varepsilon_{q_{y}}$ are independent, $f\left(\varepsilon_{q_{x}}, \varepsilon_{q_{y}}\right)=f_{\varepsilon_{q_{x}}}\left(\varepsilon_{q_{x}}\right)$ $f_{\varepsilon_{q_{y}}}\left(\varepsilon_{q_{y}}\right)$. Substituting this into the above equation, we obtain the pdf of the spatial quantization error

$$
\begin{aligned}
f_{\tilde{\varepsilon}_{q}}\left(\tilde{\varepsilon}_{q}\right)= & \frac{1}{|\cos \gamma|} \int_{-\infty}^{+\infty} f_{\varepsilon_{q_{x}}}\left(\tan \gamma\left(\frac{1}{\sin \gamma} \tilde{\varepsilon}_{q}-\tau\right)\right) \\
& \cdot f_{\varepsilon_{q_{y}}}(\tau) d \tau
\end{aligned}
$$

\section{DisPlaCEMENT ERROR}

In active vision inspection, different sensor settings are used to position the active head to obtain and inspect various dimensions of interest. If the sensor location and orientation are different from the planned sensor setting (i.e., there is sensor displacement), the same entities may be observable, but as a result of the displacement, the dimensions derived from the image will be inaccurate. The difference between the observed dimensions and the actual dimensions is defined as displacement error. The analysis in this section yields a better understanding of dimensional measurement incorporating error due to displacement of the active sensor.

\section{A. Translational and Orientational Errors in Perspective Images}

In active vision inspection, the desired positions and orientations of the head are provided to the servo control which accomplishes the sensor placement task by a sequence of movements. The horizontal and vertical displacements of the projected points on the 2-D image are in terms of the six orientation and translation parameters of the sensor setting, the focal length of the sensor, the translational and orientational errors, and the 3-D coordinates of the model.

Given the orientation and location of the sensor and the coordinates of the object, we can compute the projected image of the object as

$$
\begin{aligned}
{\left[\begin{array}{c}
x_{I} \\
y_{I} \\
z_{I} \\
c
\end{array}\right] } & =P_{\operatorname{per} Q} Q\left[\begin{array}{c}
x_{w} \\
y_{w} \\
z_{w} \\
1
\end{array}\right] \\
u & =x_{I} / c \text { and } v=y_{I} / c
\end{aligned}
$$

where $\left[x_{w}, y_{w}, z_{w}\right]^{T}$ are the coordinates of the object in the world coordinate system, $(u, v)$ are the projected coordinates 
in the image plane, $P_{\text {per }}$ is the matrix for perspective projection, and $Q$ is the transformation matrix between the world coordinates and the image coordinate system

$$
P_{\text {per }}=\left[\begin{array}{cccc}
1 & 0 & 0 & 0 \\
0 & 1 & 0 & 0 \\
0 & 0 & 1 & -\frac{1}{f} \\
0 & 0 & 0 & 1
\end{array}\right] ; \quad Q=\left[\begin{array}{cccc}
r_{11} & r_{12} & r_{13} & t_{x} \\
r_{21} & r_{22} & r_{23} & t_{y} \\
r_{31} & r_{32} & r_{33} & t_{z} \\
0 & 0 & 0 & 0
\end{array}\right]
$$

$f$ is the focal length of the sensor, $\left[r_{i j}\right]_{3 \times 3}$ is the rotation submatrix in terms of the orientation parameters and $\left[t_{k}\right]_{3 \times 1}$ is the translation submatrix in terms of the translation parameters.

If the sensor is displaced, the correct coordinates of the projected points must be computed with modification of the $Q$ matrix because the rotation and translation parameters are distorted due to displacement. Thus, a matrix $Q^{\prime}$ must be substituted for $Q$ in (4) to compensate. $Q^{\prime}$ is in terms of the three translational errors, $d x, d y, d z$, and three orientational errors, $\delta x, \delta y$, and $\delta z$, and the original translational and orientational parameters. The perspective matrix is unchanged because its only parameter, $f$, is fixed

$$
\begin{aligned}
Q^{\prime}= & Q+\Delta Q \\
= & \operatorname{Trans}\left(d_{x}, d_{y}, d_{z}\right) \operatorname{Rot}(x, \delta x) \operatorname{Rot}(y, \delta y) \\
& \cdot \operatorname{Rot}(z, \delta z) Q
\end{aligned}
$$

where Trans $(d x, d y, d z)$ is a transformation representing a translation by $d x, d y$, and $d z$. $\operatorname{Rot}(x, \delta x), \operatorname{Rot}(y, \delta y)$, and $\operatorname{Rot}(z, \delta z)$ are transformations representing a differential rotation about the $x, y$, and $z$ directions, respectively. $\Delta$ is given as

$$
\Delta=\left[\begin{array}{cccc}
0 & -\delta z & \delta y & d x \\
\delta z & 0 & -\delta x & d y \\
-\delta y & \delta x & 0 & d z \\
0 & 0 & 0 & 0
\end{array}\right]
$$

As a result, the displaced image coordinates $\left(u^{\prime}, v^{\prime}\right)$ are computed using (4)-(6) and are given as

and

$$
u^{\prime}=\frac{f(C 1+C 3 \delta y-C 2 \delta z+d x)}{f-(C 3+C 2 \delta x-C 1 \delta y+d z)},
$$

$$
v^{\prime}=\frac{f(C 2+C 1 \delta z-C 3 \delta x+d y)}{f-(C 3+C 2 \delta x-C 1 \delta y+d z)}
$$

where $C 1=r_{11} x_{w}+r_{12} y_{w}+r_{13} z_{w}+t_{x}, C 2=r_{21} x_{w}+$ $r_{22} y_{w}+r_{23} z_{w}+t_{y}$, and $C 3=r_{31} x_{w}+r_{32} y_{w}+r_{33} z_{w}+t_{z}$. The image coordinates $(u, v)$ without the displacement of the head are

$$
u=\frac{f C 1}{f-C 3}, \quad v=\frac{f C 2}{f-C 3} .
$$

The horizontal and vertical displacement errors, $\varepsilon_{\boldsymbol{d}_{u}}$ and $\varepsilon_{\boldsymbol{d}_{v}}$, are shown as (7) and (8), shown at the bottom of the page, where

$$
\begin{aligned}
\lambda_{1} & =f C 1 C 2 \quad \lambda_{2}=f\left((f-C 3) C 3-C 1^{2}\right) \\
\lambda_{3} & =f C 2(C 3-f) \\
\lambda_{4} & =f(f-C 3) \quad \lambda_{5}=0 \quad \lambda_{6}=f C 1 \\
\lambda_{7} & =0 \quad \lambda_{8}=f\left(C 2^{2}-(f-C 3) C 3\right) \quad \lambda_{9}=-f C 1 C 2 \\
\lambda_{10} & =f C 1(f-C 3) \quad \lambda_{11}=0 \quad \lambda_{12}=f(f-C 3) \\
\lambda_{13} & =f C 2 \quad \lambda_{14}=0 \quad \lambda_{15}=-C 2(f-C 3) \\
\lambda_{16} & =C 1(f-C 3) \quad \lambda_{17}=0 \quad \lambda_{18}=0 \\
\lambda_{19} & =0 \quad \lambda_{20}=f-C 3 \quad \lambda_{21}=(f-C 3)^{2} .
\end{aligned}
$$

In (8) and (9), the displacement errors are in terms of the focal length of the sensor, the 3-D world coordinates, $\left(x_{w}, y_{w}, z_{w}\right)$, the translation and orientation parameters of the sensor, and the translational and orientational errors of the active head $[d x, d y, d z, \delta x, \delta y, \delta z]$. As a result, for different 3-D points projected onto an image plane, the horizontal and vertical displacement errors on the image are not the same even if the focal length of the sensor, and the translational and orientational errors of the manipulator are the same. For instance, two points on a 3-D object, $\left(x_{1}, y_{1}, z_{1}\right)$ and $\left(x_{2}, y_{2}, z_{2}\right)$, may have unequal horizontal displacement errors, $\varepsilon_{d_{u 1}}$ and $\varepsilon_{d_{u 2}}$, and unequal vertical displacement errors, $\varepsilon_{d_{v 1}}$ and $\varepsilon_{d_{v 2}}$. This implies that the distribution of the displacement error for each projected point in an image is unique in spite of being generated by the same distribution of translational and orientational errors of the sensor.

\section{B. Probability Density Function of Displacement Errors}

Suppose that the uncertainties in translation and orientation errors are all normal distributed with zero mean [21] such that

$$
f_{a}(a)=\frac{1}{\sqrt{2 \pi} \sigma_{a}} e^{-\left(a^{2} / 2 \sigma_{a}^{2}\right)}
$$

where $a$ represents any one of the translation errors $d x, d y, d z$, or the orientation errors $\delta x, \delta y, \delta z$. Normal distributions allow simple propagation of the errors through linear relationships. If other distributions are used, more complicated propagation will be required. The normal distribution is also commonly used in error propagation for robot manipulators [21]. Since the image coordinate errors $\varepsilon_{d_{u}}$ and $\varepsilon_{d_{v}}$ are in terms of the six translation and orientation errors, we compute the pdf of these errors as follows.

$$
\begin{aligned}
& \varepsilon_{d_{u}}=u^{\prime}-u=\frac{\left[\begin{array}{llllllllllll}
\lambda_{1} & \lambda_{2} & \lambda_{3} & \lambda_{4} & \lambda_{5} & \lambda_{6} & \lambda_{7}
\end{array}\right]\left[\begin{array}{llllllll}
\delta x & \delta y & \delta z & d x & d y & d z & 1
\end{array}\right]^{T}}{\left[\begin{array}{lllllllll}
\lambda_{15} & \lambda_{16} & \lambda_{17} & \lambda_{18} & \lambda_{19} & \lambda_{20} & \lambda_{21}
\end{array}\right]\left[\begin{array}{lllllll}
\delta x & \delta y & \delta z & d x & d y & d z & 1
\end{array}\right]^{T}}
\end{aligned}
$$

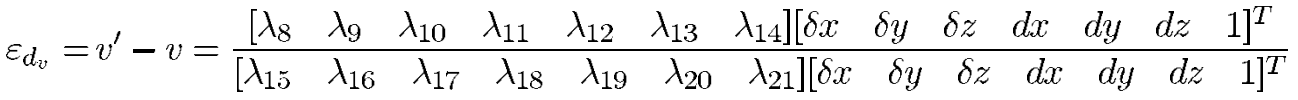


If $a=\beta_{1} a_{1}+\beta_{2} a_{2}+\cdots+\beta_{n} a_{n}+\alpha$ where $a_{i}$ are all normal distributed with zero mean and independent for $i=1,2, \cdots, n$ and $\alpha$ is a constant then $a$ is normally distributed with

mean $\mu_{a}=\alpha$ and variance $\sigma_{a}^{2}=\beta_{1}^{2} \sigma_{a_{1}}^{2}+\beta_{2}^{2} \sigma_{a_{2}}^{2}+\cdots+\beta_{n}^{2} \sigma_{a_{n}}^{2}$.

From (7) and (8), the horizontal and vertical displacement errors, $\varepsilon_{d_{u}}$ and $\varepsilon_{d_{v}}$, are rational functions where both the numerator and the denominator are in terms of three rotational errors and three translational errors of the active head and a constant.

Let

$$
\varepsilon_{d_{u}}=\frac{\zeta}{\chi} \quad \text { and } \quad \varepsilon_{d_{v}}=\frac{\xi}{\chi}
$$

where

$$
\begin{aligned}
\zeta= & {\left[\begin{array}{lllllll}
\lambda_{1} & \lambda_{2} & \lambda_{3} & \lambda_{4} & \lambda_{5} & \lambda_{6} & \lambda_{7}
\end{array}\right] } \\
& \cdot\left[\begin{array}{lllllll}
\delta x & \delta y & \delta z & d x & d y & d z & 1
\end{array}\right] \\
\xi= & {\left[\begin{array}{llllllll}
\lambda_{8} & \lambda_{9} & \lambda_{10} & \lambda_{11} & \lambda_{12} & \lambda_{13} & \lambda_{14}
\end{array}\right] } \\
& \cdot\left[\begin{array}{lllllll}
\delta x & \delta y & \delta z & d x & d y & d z & 1
\end{array}\right] \\
\chi= & {\left[\begin{array}{lllllll}
\lambda_{15} & \lambda_{16} & \lambda_{17} & \lambda_{18} & \lambda_{19} & \lambda_{20} & \lambda_{21}
\end{array}\right] } \\
& \cdot\left[\begin{array}{lllllll}
\delta x & \delta y & \delta z & d x & d y & d z & 1
\end{array}\right] .
\end{aligned}
$$

As described above, the numerators and the denominator are normally distributed where $\mu_{\zeta}=\lambda_{7}=0, \sigma_{\zeta}^{2}=\lambda_{1}^{2} \sigma_{\delta x}^{2}+$ $\lambda_{2}^{2} \sigma_{\delta y}^{2}+\lambda_{3}^{2} \sigma_{\delta z}^{2}+\lambda_{4}^{2} \sigma_{d x}^{2}+\lambda_{5}^{2} \sigma_{d y}^{2}+\lambda_{6}^{2} \sigma_{d z}^{2}, \mu_{\xi}=\lambda_{14}=0$ $\sigma_{\xi}^{2}=\lambda_{8}^{2} \sigma_{\delta x}^{2}+\lambda_{9}^{2} \sigma_{\delta y}^{2}+\lambda_{10} \sigma_{\delta z}^{2}+\lambda_{11} \sigma_{d x}^{2}+\lambda_{12}^{2} \sigma_{d y}^{2}+\lambda_{13}^{2} \sigma_{d z}^{2}$, $\mu_{\chi}=\lambda_{21}=(f-C 3)^{2}$, and $\sigma_{\chi}^{2}=\lambda_{15}^{2} \sigma_{\delta x}^{2}+\lambda_{16}^{2} \sigma_{\delta y}^{2}+\lambda_{17}^{2} \sigma_{\delta z}^{2}+$ $\lambda_{18}^{2} \sigma_{d x}^{2}+\lambda_{19}^{2} \sigma_{d y}^{2}+\lambda_{20}^{2} \sigma_{d z}^{2}$.

Since $\zeta, \xi$, and $\chi$ are all in terms of the same translational and rotational errors, they are dependent on each other. To find the pdf of $\varepsilon_{d_{u}}$ and $\varepsilon_{d_{v}}$, the correlation coefficients of $\xi$ and $\chi$, and $\zeta$ and $\chi$ are used.

Let

$$
\begin{aligned}
& g_{1}\left(\varepsilon_{\boldsymbol{d}_{u}}\right)=\sigma_{\zeta}^{2}-2 r_{\zeta, \chi} \sigma_{\zeta} \sigma_{\chi} \varepsilon_{\boldsymbol{d}_{u}}+\sigma_{\chi}^{2} \varepsilon_{\boldsymbol{d}_{u}}^{2} \\
& g_{2}\left(\varepsilon_{\boldsymbol{d}_{u}}\right)=\sigma_{\zeta}-r_{\zeta \chi} \sigma_{\chi} \varepsilon_{\boldsymbol{d}_{u}} \\
& h_{1}\left(\varepsilon_{\boldsymbol{d}_{v}}\right)=\sigma_{\xi}^{2}-2 r_{\xi, \chi} \sigma_{\chi} \varepsilon_{\boldsymbol{d}_{v}} \sigma_{\xi}+\sigma_{\chi}^{2} \varepsilon_{\boldsymbol{d}_{v}}^{2} \\
& h_{2}\left(\varepsilon_{\boldsymbol{d}_{v}}\right)=\sigma_{\xi}-r_{\xi, \chi} \sigma_{\chi} \varepsilon_{\boldsymbol{d}_{v}} .
\end{aligned}
$$

If $f=C 3$, and either $\operatorname{Cov}(\zeta, \chi)=0$ or $\operatorname{Cov}(\xi, \chi)=0$, then $r_{\zeta, \chi}=\mu_{\chi}=0$ or $r_{\xi, \chi}=\mu_{\chi}=0$, respectively. The pdf of the horizontal and vertical errors of image coordinates are then

and

$$
f_{\varepsilon_{d_{u}}}\left(\varepsilon_{d_{u}}\right)=\frac{\sigma_{\zeta} \sigma_{\chi}}{\pi g_{1}\left(\varepsilon_{d_{u}}\right)}
$$

$$
f_{\varepsilon_{d_{v}}}\left(\varepsilon_{d_{v}}\right)=\frac{\sigma_{\xi} \sigma_{\chi}}{\pi h_{1}\left(\varepsilon_{d_{v}}\right)} .
$$

If $f=C 3, \mu_{\chi}=0$. The pdf's of the horizontal and vertical errors of image coordinates are then

and

$$
f_{\varepsilon_{d_{u}}}\left(\varepsilon_{\boldsymbol{d}_{u}}\right)=\frac{\sigma_{\zeta} \sigma_{\chi} \sqrt{1-r_{\zeta, \chi}^{2}}}{\pi g_{1}\left(\varepsilon_{\boldsymbol{d}_{u}}\right)}
$$

$$
f_{\varepsilon_{d_{v}}}\left(\varepsilon_{\boldsymbol{d}_{v}}\right)=\frac{\sigma_{\xi} \sigma_{\chi} \sqrt{1-r_{\xi, \chi}^{2}}}{\pi h_{1}\left(\varepsilon_{\boldsymbol{d}_{v}}\right)} .
$$

If $\operatorname{Cov}(\zeta, \chi)=0$ or $\operatorname{Cov}(\xi, \chi)=0, r_{\zeta, \chi}$ or $r_{\xi, \chi}$ is zero, respectively. In this case, the pdf's of the horizontal and vertical errors of image coordinates are then

$$
\begin{aligned}
f_{\varepsilon_{d_{u}}}\left(\varepsilon_{\boldsymbol{d}_{u}}\right)= & \frac{\sigma_{\zeta} \sigma_{\chi}}{\pi g_{1}\left(\varepsilon_{\boldsymbol{d}_{u}}\right)} \exp \left(-\frac{\mu_{\chi}^{2}}{2 g_{1}\left(\varepsilon_{\boldsymbol{d}_{u}}\right)}\left(\varepsilon_{\boldsymbol{d}_{u}}^{2}+\frac{\sigma_{\zeta}^{2}}{\sigma_{\chi}^{2}}\right)\right) \\
& +\frac{\mu_{\chi} \sigma_{\zeta}^{2}}{\sqrt{2 \pi} g_{1}\left(\varepsilon_{\boldsymbol{d}_{u}}\right)^{3 / 2}} \exp \left(-\frac{\mu_{\chi}^{2} \varepsilon_{\boldsymbol{d}_{u}}^{2}}{2 g_{1}\left(\varepsilon_{\boldsymbol{d}_{u}}\right)}\right) \\
& \cdot \operatorname{erf}\left(\frac{\mu_{\chi} \sigma_{\zeta}}{\left.\sigma_{\chi} \sqrt{2 g_{1}\left(\varepsilon_{d_{u}}\right)}\right)}\right) \\
f_{\varepsilon_{d_{v}}}\left(\varepsilon_{\boldsymbol{d}_{v}}\right)= & \frac{\sigma_{\xi} \sigma_{\chi}}{\pi h_{1}\left(\varepsilon_{\left.\boldsymbol{d}_{v}\right)}\right)} \exp \left(-\frac{\mu_{\chi}^{2}}{2 h_{1}\left(\varepsilon_{\boldsymbol{d}_{v}}\right)}\left(\varepsilon_{d_{v}}^{2}+\frac{\sigma_{\xi}^{2}}{\sigma_{\chi}^{2}}\right)\right) \\
& +\frac{\mu_{\chi} \sigma_{\xi}^{2}}{\sqrt{2 \pi} h_{1}\left(\varepsilon_{d_{v}}\right)^{3 / 2}} \exp \left(-\frac{\mu_{\chi}^{2} \varepsilon_{d_{v}}^{2}}{2 h_{1}\left(\varepsilon_{\boldsymbol{d}_{v}}\right)}\right) \\
& \cdot \operatorname{erf}\left(\frac{\mu_{\chi} \sigma_{\xi}}{\sigma_{\chi} \sqrt{2 h_{1}\left(\varepsilon_{d_{v}}\right)}}\right) .
\end{aligned}
$$

Otherwise

$$
\begin{aligned}
& f_{\varepsilon_{d_{u}}}\left(\varepsilon_{d_{u}}\right) \\
& =\frac{\sigma_{\zeta} \sigma_{\chi} \sqrt{1-r_{\zeta \chi}^{2}}}{\pi g_{1}\left(\varepsilon_{d_{u}}\right)} \exp \left(-\frac{\mu_{\chi}^{2}}{2 g_{1}\left(\varepsilon_{d_{u}}\right)}\left(\varepsilon_{u}^{2}+\frac{g_{2}\left(\varepsilon_{d_{u}}\right)^{2}}{\sigma_{\chi}^{2}}\right)\right) \\
& +\frac{g_{2}\left(\varepsilon_{\boldsymbol{d}_{u}}\right) \mu_{\chi} \sigma_{\zeta}}{\sqrt{2 \pi} g_{1}\left(\varepsilon_{\boldsymbol{d}_{u}}\right)^{3 / 2}} \exp \left(-\frac{\mu_{\chi}^{2} \varepsilon_{\boldsymbol{d}_{u}}^{2}}{2 g_{1}\left(\varepsilon_{\boldsymbol{d}_{u}}\right)}\right) \\
& \cdot \operatorname{erf}\left(\frac{g_{2}\left(\varepsilon_{d_{u}}\right) \mu_{\chi}}{\sigma_{\chi} \sqrt{2\left(1-r_{\zeta \chi}^{2}\right) g_{1}\left(\varepsilon_{d_{u}}\right)}}\right) \\
& f_{\varepsilon_{d_{v}}}\left(\varepsilon_{d_{v}}\right) \\
& \begin{aligned}
= & \frac{\sigma_{\xi} \sigma_{\chi} \sqrt{1-r_{\xi, \chi}^{2}}}{\pi h_{1}\left(\varepsilon_{d_{v}}\right)} \exp \left(-\frac{\mu_{\chi}^{2}}{2 h_{1}\left(\varepsilon_{d_{v}}\right)}\left(\varepsilon_{d_{v}}^{2}+\frac{h_{2}\left(\varepsilon_{d_{v}}\right)^{2}}{\sigma_{\chi}^{2}}\right)\right) \\
& +\frac{h_{2}\left(\varepsilon_{d_{v}}\right) \mu_{\chi} \sigma_{\xi}}{\sqrt{2 \pi} h_{1}\left(\varepsilon_{d_{v}}\right)^{3 / 2}} \exp \left(-\frac{\mu_{\chi}^{2} \varepsilon_{\boldsymbol{d}_{v}}^{2}}{2 h_{1}\left(\varepsilon_{d_{v}}\right)}\right) \\
& \cdot \operatorname{erf}\left(\frac{h_{2}\left(\varepsilon_{d_{v}}\right) \mu_{\chi}}{\sigma_{\chi} \sqrt{2\left(1-r_{\xi \chi}^{2}\right) g_{1}\left(\varepsilon_{d_{v}}\right)}}\right) .
\end{aligned}
\end{aligned}
$$

1) Characteristics of the Probability Density Function of Displacement Errors: In order to study the effect of the displacement errors on active vision inspection, the characteristics of their pdf's must be understood. The last section derived the pdf's for the horizontal and vertical errors in the image coordinates of a point based on the displacement errors. The parameters in these functions are the focal length of the camera, $f$, and the coordinates of the ideal projection point in the camera coordinate system, $(C 1, C 2, C 3)$. In this section, the sensitivity of the pdf's to those parameters is explored through several examples.

The pdf's of the horizontal and vertical displacements errors, (11) and (12), are derived based on the mean, variance, and correlation coefficients of the numerator and denominator of 


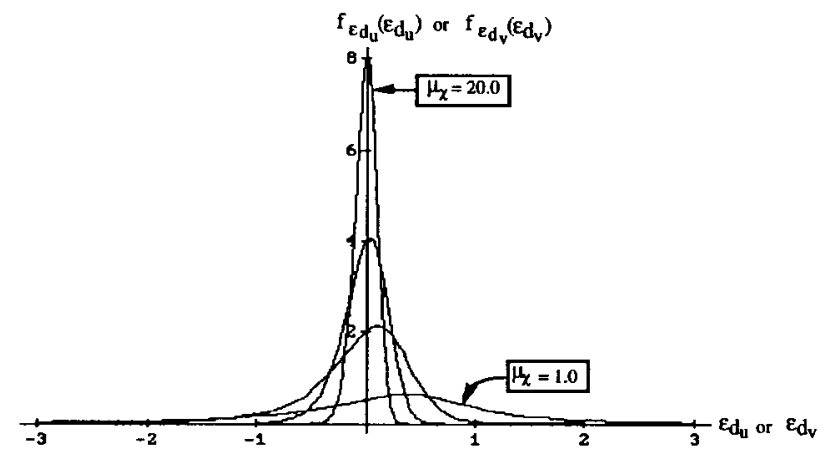

Fig. 3. The pdf's of the displacement errors due to displacement of the end-effectors in horizontal or vertical directions, $f_{\varepsilon_{d u}}\left(\varepsilon_{d_{u}}\right.$ or $f_{\varepsilon_{d v}}\left(\varepsilon_{d_{v}}\right)$, where $\mu_{\chi}=1.0,5.0,10.0$, or 20.0, $\sigma_{\zeta}=\sigma_{\xi}=\sigma_{\chi}=2.0$, and $r_{\zeta, \chi}=r_{\xi, \chi}=0.5$

the errors given in (7) and (8). Both the numerator and denominator of (7) and (8) are normally distributed. The means $\left(\mu_{\zeta}, \mu_{\xi}, \mu_{\chi}\right)$, variances $\left(\sigma_{\zeta}^{2}, \sigma_{\xi}^{2}, \sigma_{\chi}^{2}\right)$, and correlation coefficients $\left(r_{\zeta \chi}, r_{\xi, \chi}\right)$ all depend on $f, C 1, C 2, C 3$, and the variance of the translational and orientational errors of the active head. Given the pdf's of the translational and orientational errors of the active head, the focal length, and the projection of the model point in the sensor coordinates, one can determine the mean, variance and the correlation coefficients of the numerator and the denominator. Considering one of these parameters at a time

i) $\mu_{\zeta}, \mu_{\xi}, \mu_{\chi}$

ii) $r_{\zeta, \chi}, r_{\xi, \chi}$

we will explore and analyze the properties of the pdf of the horizontal displacement errors.

\section{i) $\mu_{\zeta}, \mu_{\xi}$, and $\mu_{\chi}$}

Since $\lambda_{7}$ and $\lambda_{14}$ are zero, the means of the numerator for the errors, $\mu_{\zeta}$ and $\mu_{\xi}$, are both zero. The mean of the denominators for the errors, $\mu_{\chi}$, is equal to $\lambda_{21}$ which is $(f-C 3)^{2} \cdot \mu_{\chi}$ increases as $|f-C 3|$ increases. $f$ is the focal length of the camera and $C 3$ is the distance between the model point and the image plane. $f$ is based on the lens of the sensor and it may be assumed to be fixed, however, $C 3$ depends on the distance between the object and the sensor. Thus, $\mu_{\chi}$ increases as the orthogonal distance between the inspected object and the sensor increases. Fig. 3 shows the pdf of the horizontal and vertical displacement errors with $\mu_{\chi}$ equal to $1.0,5.0,10.0$, and 20.0. The variances, $\sigma_{\zeta}^{2}, \sigma_{\xi}^{2}, \sigma_{\chi}^{2}$, are 2.0 , and the correlation coefficients, $r_{\zeta, \chi}, r_{\xi, \chi}$, are 0.5. This figure shows that the mode is closer to zero, its probability density value increases, and the shape of the curve is narrower when $\mu_{\chi}$ increases. As a result, the probability of the projected point on the 2-D image having large horizontal or vertical displacement errors decreases if the inspected object is farther from the sensor.

\section{ii) $r_{\zeta, \chi}$ and $r_{\xi, \chi}$}

The distributions of the numerators, $\zeta$ and $\xi$, and the denominators, $\chi$, of the displacement errors are normal and dependent as discussed in Section III-B. The covariances of

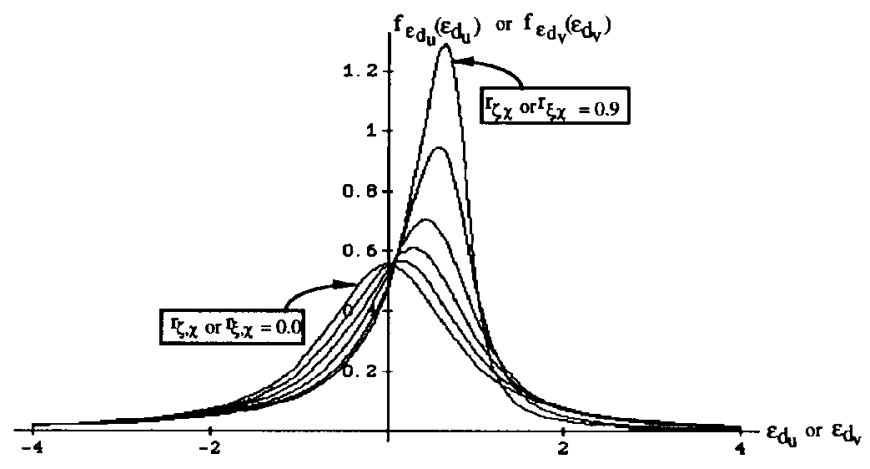

(a)

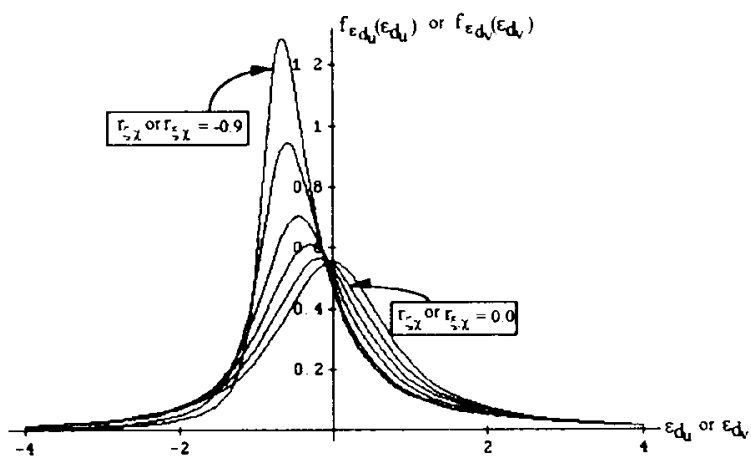

(b)

Fig. 4. The pdf's of the displacement errors affected by the displacement of the end-effectors in horizontal or vertical directions, $f_{\varepsilon_{d_{u}}}\left(\varepsilon_{d_{u}}\right.$ or $f_{\varepsilon_{d_{v}}}\left(\varepsilon_{d_{v}}\right.$, (a) where $\mu_{\chi}=2.0, \sigma=\sigma=1.0, \sigma_{\chi}=1.0$, and $r_{\zeta, \chi}=r_{\xi, \chi}=0.0$, $0.2,0.4,0.6,0.8,0.9$, (b) where $\mu_{\chi}=2.0, \sigma=\sigma=1.0, \sigma_{\chi}=1.0$, and $r_{\zeta, \chi}=r_{\xi, \chi}=-0.9,-0.8,-0.6,-0.4,-0.2,0.0$.

the numerator and denominator measure their dependence. Let $\sigma_{i}^{2}=\sigma_{\delta x}^{2}=\sigma_{\delta y}^{2}=\sigma_{\delta z}^{2}$ and $\sigma_{j}^{2}=\sigma_{d x}^{2}=\sigma_{d y}^{2}=\sigma_{d z}^{2}$ because the variances of the orientational errors about three orthogonal axis are approximately equal and the variances of the translational errors along each direction are also approximately equal. The covariance of $\zeta$ and $\chi$ and the covariance of $\xi$ and $\chi$ are calculated as

$$
\begin{aligned}
\operatorname{Cov}(\zeta, \chi)= & f C 1(f-C 3)\left(\left(f C 3-C 1^{2}-C 2^{2}-C 3^{2}\right)\right. \\
& \left.\cdot \sigma_{i}^{2}+\sigma_{j}^{2}\right) \\
\operatorname{Cov}(\xi, \chi)= & f C 2(f-C 3)\left(\left(f C 3-C 1^{2}-C 2^{2}-C 3^{2}\right)\right. \\
& \left.\cdot \sigma_{i}^{2}+\sigma_{j}^{2}\right) .
\end{aligned}
$$

If $\operatorname{Cov}(\zeta, \chi)>0, \zeta$ and $\chi$ are positively correlated, otherwise, they are negatively correlated. A similar situation holds for $\xi$ and $\chi$. Fig. 4(a) and (b) show the effects of the correlation coefficient of the numerator and denominator on the pdf of horizontal and vertical displacement errors of projected points with $r_{\zeta, \chi}, r_{\zeta, \chi}$ varying from $-0.9-+0.9, \mu_{\chi}=2.0$, and $\sigma_{\zeta}^{2}, \sigma_{\xi}^{2}, \sigma_{\chi}^{2}=1.0$. These figures show that the mode is positive when the correlation coefficient is positive and the mode is negative when the correlation coefficient is negative. The mode is zero when the correlation coefficient is zero. As the correlation coefficient increases from zero, the mode increases and the probability density value of the mode increases. Similarly, as the correlation coefficient decreases from zero, the mode decreases and the probability density value of the mode increases. The absolute value of the probability density 
value of the mode is minimum when the correlation coefficient is zero. Thus, the likelihood of having more positive errors or more negative errors depends on the sign of $\operatorname{Cov}(\zeta, \chi)$ and $\operatorname{Cov}(\xi, \chi)$ and the probability of having large errors depends on the absolute value of $\operatorname{Cov}(\zeta, \chi)$ and $\operatorname{Cov}(\xi, \chi)$.

Since the correlation coefficient is equal to the covariance between the numerator and denominator divided by the product of their standard deviations, the sign of the correlation coefficient and the mode of the pdf depends on the sign of the covariance between the numerator and the denominator. From (13) and (14), $\operatorname{Cov}(\zeta, \chi)$ and $\operatorname{Cov}(\xi, \chi)$ are in terms of $f, C 1, C 2, C 3, \sigma_{i}^{2}$, and $\sigma_{j}^{2}$. Indeed, $\operatorname{Cov}(\zeta, \chi)=\operatorname{Cov}(\xi, \chi)$ when $C 1=C 2$ for all values of $C 3 . f$ and $C 3$ are positive and $f$ is small compared to $C 3$, therefore, $(f-C 3)$ and $\left(f C 3-C 1^{2}-C 2^{2}-C 3^{2}\right)$ are always negative. As a result, if $\left(f C 3-C 1^{2}-C 2^{2}-C 3^{2}\right) \sigma_{i}^{2}+\sigma_{j}^{2} \leq 0, \operatorname{Cov}(\zeta, \chi)$ is an increasing function of $C 1$ and is 0 when $C 1=0$. Similarly, if $\left(f C 3-C 1^{2}-C 2^{2}-C 3^{2}\right) \sigma_{i}^{2}+\sigma_{j}^{2} \leq 0, \operatorname{Cov}(\xi, \chi)$ is an increasing function of $C 2$ and is 0 when $C 2=0$. (fC3$\left.C 1^{2}-C 2^{2}-C 3^{2}\right) \sigma_{i}^{2}+\sigma_{j}^{2} \leq 0$ is typically true because $C 3$ is much larger than $\sigma_{i}$ and $\sigma_{j}$. This analysis shows that the probability of large horizontal displacement errors increases when the horizontal distance between the inspected object and the optical axis of the sensor increases. A similar situation holds for the vertical displacement errors.

\section{Displacement Errors in Dimensional Measurement}

The displacement errors in projected points result in errors in the measured area of a surface, the measured curvature of an arc or the measured length of a line segment. These features are composed of the projection of the corresponding points from the 3-D model. In this section, we will investigate the displacement errors introduced in the dimension measurement of linear segments.

The coordinates of the end-points of a linear segment in an image are $(u 1, v 1)$ and $(u 2, v 2)$. The length of the line segment is the distance between the end-points. For fixed translational and orientational errors of the sensor, the values of horizontal and vertical displacement errors of $u 1$ and $u 2$ are not identical; similarly, the displacement errors of $v 1$ and $v 2$ are not identical.

Let $(u 1, v 1)$ correspond to the projection of model point $(x 1, y 1, z 1)$ and $(u 2, v 2)$ correspond to the projection of model point $(x 2, y 2, z 2)$. Using (7) and (8), the horizontal and vertical displacement errors can be computed.

The displacement error in the dimension of the line segment is composed of two components, the horizontal component $\varepsilon_{d_{x}}$ and the vertical component $\varepsilon_{d_{y}}$. The horizontal component, $\varepsilon_{d_{x}}$, is equal to $\varepsilon_{d_{u_{1}}}-\varepsilon_{d_{u_{2}}}$, and similarly, the vertical component, $\varepsilon_{d_{y}}$, is equal to $\varepsilon_{d_{v_{1}}}-\varepsilon_{d_{v_{2}}}$. The pdf's of $\varepsilon_{d_{u_{1}}}, \varepsilon_{d_{u_{2}}}, \varepsilon_{d_{v_{1}}}$, and $\varepsilon_{d_{v_{2}}}$ are given in (11) and (12). The pdf of $\varepsilon_{d_{x}}$ and $\varepsilon_{d_{y}}$ can be obtained by integrating the pdf's of $\varepsilon_{d_{u_{1}}}$, and $\varepsilon_{d_{u_{2}}}$, and $\varepsilon_{d_{v_{1}}}$, and $\varepsilon_{d_{v_{2}}}$, respectively. The correlations between $\varepsilon_{d_{u_{1}}}$, and $\varepsilon_{d_{u_{2}}}$, and $\varepsilon_{d_{v_{1}}}$, and $\varepsilon_{d_{v_{2}}}$ are investigated by simulations. The result shows that $\varepsilon_{d_{u_{1}}}$, and $\varepsilon_{d_{u_{2}}}$, and $\varepsilon_{d_{v_{1}}}$, and $\varepsilon_{d_{v_{2}}}$ are positively correlated, therefore, $\operatorname{Cov}\left(\varepsilon_{d_{u_{1}}}, \varepsilon_{d_{u_{2}}}\right)$ and $\operatorname{Cov}\left(\varepsilon_{d_{v_{1}}}, \varepsilon_{d_{v_{2}}}\right)$ are positive. That means, the assumption of independence between $\varepsilon_{d_{u_{1}}}$, and $\varepsilon_{d_{u_{2}}}$, and $\varepsilon_{d_{v_{1}}}$, and $\varepsilon_{d_{v_{2}}}$ causes an overestimation of the variance in the errors. The pdf's of $\varepsilon_{d_{x}}$ and $\varepsilon_{d_{y}}$, obtained by assuming independence between $\varepsilon_{d_{u_{1}}}$, and $\varepsilon_{d_{u_{2}}}$, and $\varepsilon_{d_{v_{1}}}$, and $\varepsilon_{d_{v_{2}}}$, are computed by the convolution of the pdf's of $\varepsilon_{d_{u_{1}}}$ and $-\varepsilon_{d_{u_{2}}}$, and $\varepsilon_{d_{v_{1}}}$ and $-\varepsilon_{d_{v_{2}}}$, respectively

$$
\begin{aligned}
& f_{\varepsilon_{d_{x}}}\left(\varepsilon_{d_{x}}\right)=\int_{-\infty}^{\infty} f_{\varepsilon_{d_{u_{1}}}}\left(\varepsilon_{d_{x}}+\tau\right) f_{\varepsilon_{d_{u_{2}}}}(\tau) d \tau \text { and } \\
& f_{\varepsilon_{d_{y}}}\left(\varepsilon_{d_{y}}\right)=\int_{-\infty}^{\infty} f_{\varepsilon_{d_{v_{1}}}}\left(\varepsilon_{d_{y}}+\tau\right) f_{\varepsilon_{d_{v_{2}}}}(\tau) d \tau .
\end{aligned}
$$

Although $\varepsilon_{d_{u_{1}}}$, and $\varepsilon_{d_{u_{2}}}$, and $\varepsilon_{d_{v_{1}}}$, and $\varepsilon_{d_{v_{2}}}$ are not independent, the density function obtained by this assumption bounds the variability of the actual density function and is easy to compute.

Similar to the geometric approximation used for the spatial quantization error, the 2-D displacement error, $\varepsilon_{d}$, in the dimension of a linear segment with an angle of $\gamma$ between the segment and the horizontal axis of the image can be expressed in terms of the horizontal and vertical components of dimensioning errors due to displacement and the angle

$$
\tilde{\varepsilon}_{d}=\cos \gamma \varepsilon_{d_{x}}+\sin \gamma \varepsilon_{d_{y}} .
$$

The pdf of the dimensioning error due to the displacement of the sensor can be expressed in terms of the pdf's of the component dimensioning error and the angle, $\gamma$, in the following open form:

$$
\begin{aligned}
f_{\tilde{\varepsilon}_{d}}\left(\tilde{\varepsilon}_{d}\right)= & \frac{1}{|\cos \gamma|} \int_{-\infty}^{\infty} f_{\varepsilon_{d_{x}}}\left(\tan \gamma\left(\frac{1}{\sin \gamma} \tilde{\varepsilon}_{d}-\tau\right)\right) \\
& \cdot f_{\varepsilon_{d_{y}}}(\tau) d \tau .
\end{aligned}
$$

\section{The Total Error}

Displacement is independent of the resolution of the sensor and, consequently, independent of the quantization error. Thus, the total inspection error, $\varepsilon_{i}$, is the sum of the quantization error, $\varepsilon_{q}$, and the displacement error, $\varepsilon_{d}$

$$
\varepsilon_{i}=\varepsilon_{q}+\varepsilon_{d}
$$

The pdf of the quantization error and the displacement error, $f_{\varepsilon_{q}}\left(\varepsilon_{q}\right)$ and $f_{\varepsilon_{d}}\left(\varepsilon_{d}\right)$, are given in (3) and (17). Based on (18), the pdf of the total error is computed from the convolution of the pdf's of the quantization and displacement errors

$$
\begin{gathered}
f_{\varepsilon_{i}}\left(\varepsilon_{i}\right)=\int_{-\infty}^{\infty} f_{\varepsilon_{q}}\left(\varepsilon_{i}-\tau\right) f_{\varepsilon_{d}}(\tau) d \tau . \\
\text { IV. PLANNING ACCURACY IN } \\
\text { INSPECTION OF LINEAR DIMENSIONS }
\end{gathered}
$$

\section{PlanNing ACCURACY IN}

Accuracy in active vision inspection can be improved by careful choice of the sensor settings for each inspected dimension. A sensor setting determines the location and view direction where an active sensor may be placed to observe one or more objects which contain one or more topologic entities whose dimensions are to be measured. A sensor arrangement, $S=\left\{x_{1}, x_{2}, \cdots, x_{n}\right\}$, has $n$ sensor settings $x_{1}$ to $x_{n} . x_{i}$ is a sensor setting which is an ordered triple $\left(v_{i}, d_{i}, o_{i}\right)$ consisting 
of a sensor location $v_{i}$, a sensor view direction $d_{i}$, and a set of observable segments (features) $o_{i}$ from the given setting. The same edge segment of a part (model) can be observed using many sensor settings. Although a dimensional attribute, such as the width of a slot may be observable from different sensor settings, the utility of each sensor setting is not identical. However, because more than one entity (feature) can be captured on each image, it is usually desirable to perform several dimensional inspections from a single sensor setting in order to minimize the sensing operations and the data processing. Thus, simplistic techniques based on orthogonal direction and minimal distance are not viable methods in this case [11], [23]. Instead, it is necessary to evaluate the accuracy attainable from the sensor settings, and ensure that the potential errors in all dimensional measurements from each setting are acceptable for verification of required (part) tolerances as indicated in the design. Hence, an analysis of expected accuracy of dimensional inspections in terms of the sensor setting parameters and sensor resolution is necessary in this case.

The inspection accuracy in dimensioning a linear segment can be defined as

$$
\text { Accuracy }=1-\frac{|\varepsilon|}{L}
$$

where $L$ is the image (or projected) length of the segment. This representation can be used to analyze the utility of different sensor settings by evaluating the probability for a dimensioning accuracy to be within a particular tolerance. $L$ can be found in terms of the angle between the segment and the direction of the camera axis, $\beta$, the translational distances between the camera and the midpoint of the segment, $t_{x}, t_{y}, t_{z}$ (where $t_{x}, t_{y}$ are along the horizontal and vertical image plane axes, and $t_{z}$ is along the sensor view direction), and the model length of the segment in three dimensions, $L_{w}$ as shown in Fig. 5(a)

$$
\begin{aligned}
& L\left(L_{w}, \beta, t_{x}, t_{y}, t_{z}, f\right) \\
& \quad=\frac{L_{w} f \sqrt{t_{x}^{2} \cos ^{2} \beta+\left(\left(f+t_{z}\right) \sin \beta-t_{y} \cos \beta\right)^{2}}}{\left(f+t_{z}\right)^{2}-1 / 4 L_{w}^{2} \cos ^{2} \beta} .
\end{aligned}
$$

When the camera is pointing at the center of the segment (focal axis meets the midpoint), $t_{x}$ and $t_{y}$ are both equal to zero. In this case, the above equation can be simplified to be only in terms of $\beta, L_{w}, f$, and the distance $d$ as shown in Fig. 5(b)

$$
L\left(L_{w}, \beta, d, f\right)=L_{w} f \sin \beta \frac{d+f}{(d+f)^{2}+1 / 4 L_{w}^{2} \cos ^{2} \beta} .
$$

We obtained expressions for the pdf of $\varepsilon$ in terms of the sensor and the sensor setting parameters in previous sections. With both $L$ and the error pdf expressed in terms of these parameters, we can compute the likelihood of achieving a certain accuracy level from particular sensor settings. Comparing the variance of accuracy computed from (20) with the tolerance specified, we can determine the dimensional inspection capability for given sensor settings. Thus, given that the accuracy tolerance is $[1-T, 1]$ where $0 \leq T \leq 1$ for a dimension to be inspected, we can compute the likelihood

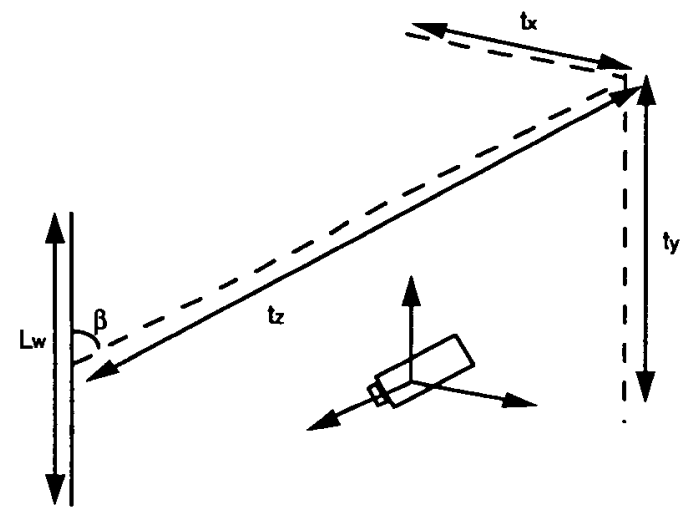

(a)

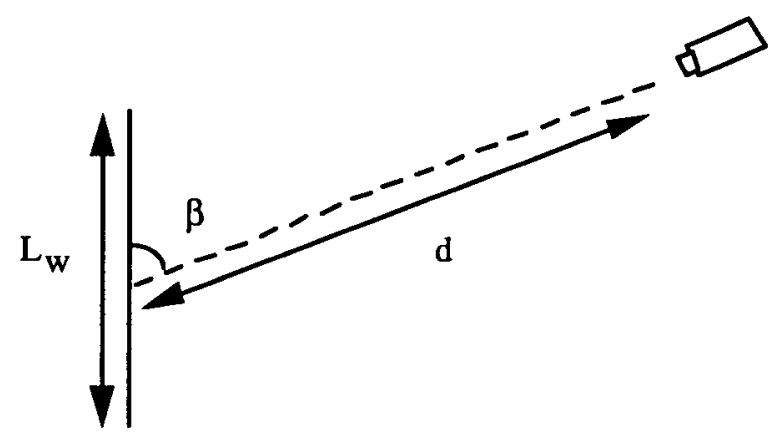

(b)

Fig. 5. (a) Line of length $L_{w}$ is projected onto the camera image plane. (a) The angle between the line and the direction of the camera is $\beta$ and the translation from the center of the line to the camera in three orthogonal direction is $t_{x}, t_{y}$, and $t_{z}$. (b) Angle between the line and the direction of the camera is $\beta$ and the distance from the camera to the center of the line is $d$.

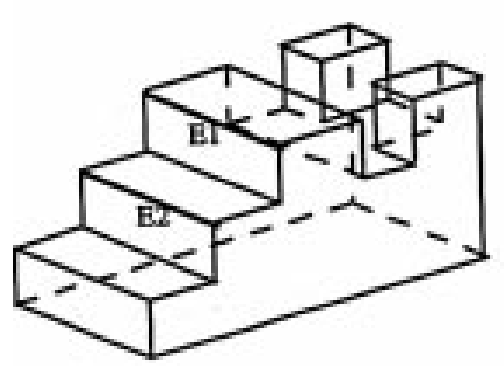

Fig. 6. An object with two slots and two steps. The dimension E2 is used in the experiment.

that the achieved accuracy is within this tolerance range by computing $\operatorname{Pr}\{$ Accuracy $\geq 1-T\}$. For example, if the likelihood is greater than a certain threshold, $T h$ where 0 $\leq T h \leq 1$, the sensor setting may be considered acceptable for inspecting the corresponding dimension, and if the probability is less, the view direction of the sensor and/or the sensor location can be changed [one method for this may be based on (21) and (22)]. Changing the angle $\beta$, and/or the distance $d$ changes the achievable accuracy. As a result, we can more effectively integrate them in our dimensional inspection strategy, and better understand their quantitative nature. This could be helpful in determining sensor settings which are better capable to dimensionally inspect part (model) attributes with a desired accuracy. 


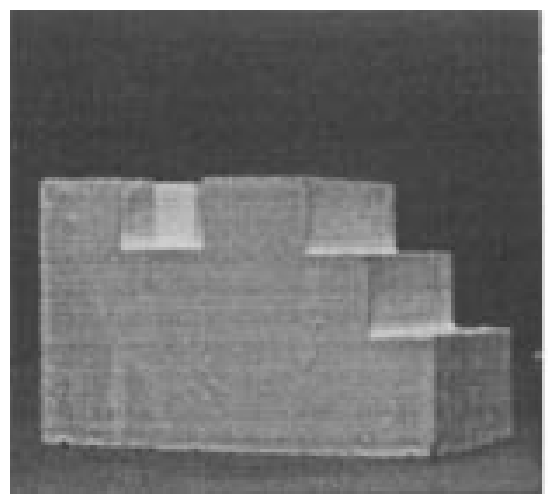

(a)

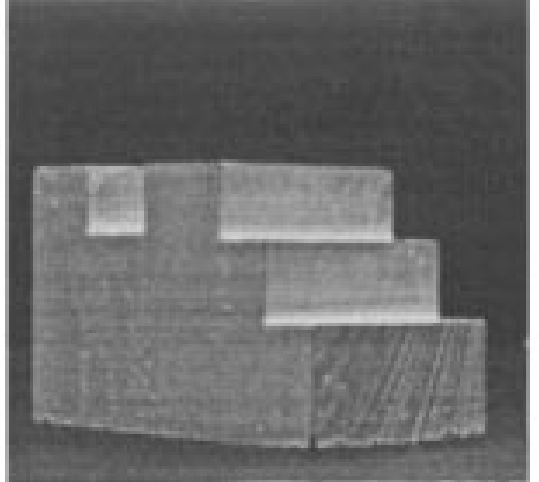

(b)

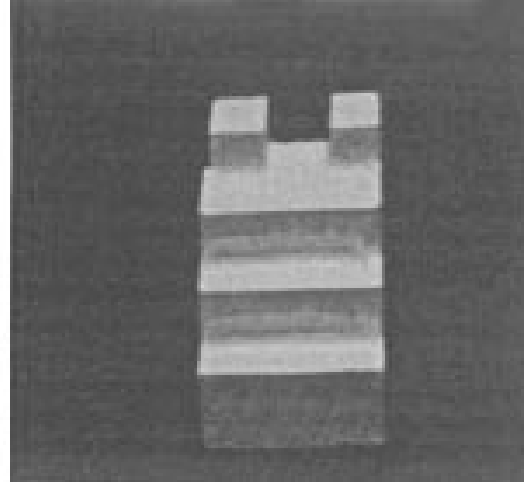

(c)

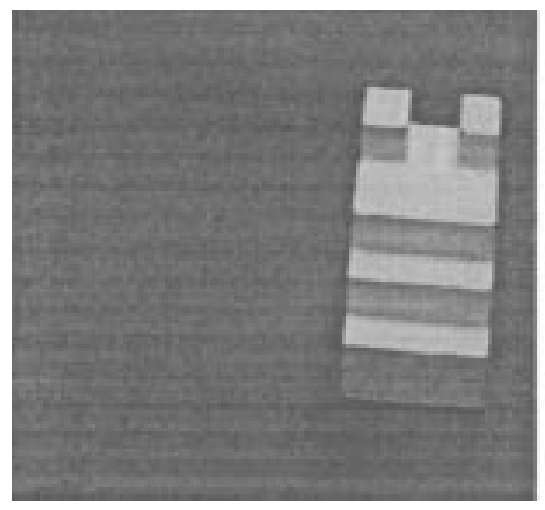

(d)

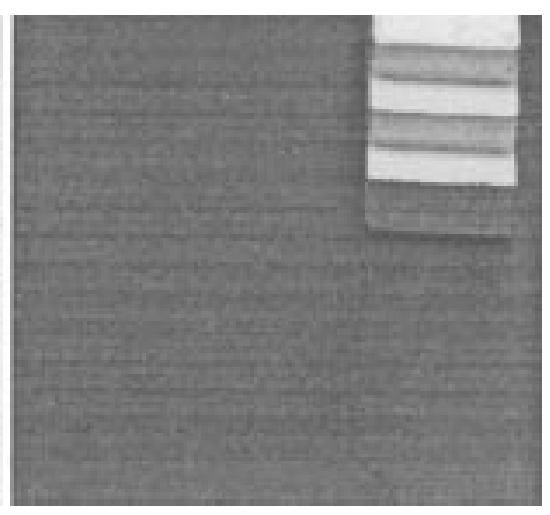

(e)

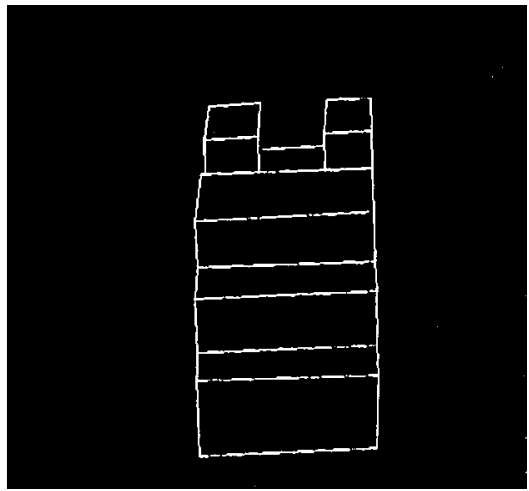

(h)

$(\mathrm{g})$

(f)
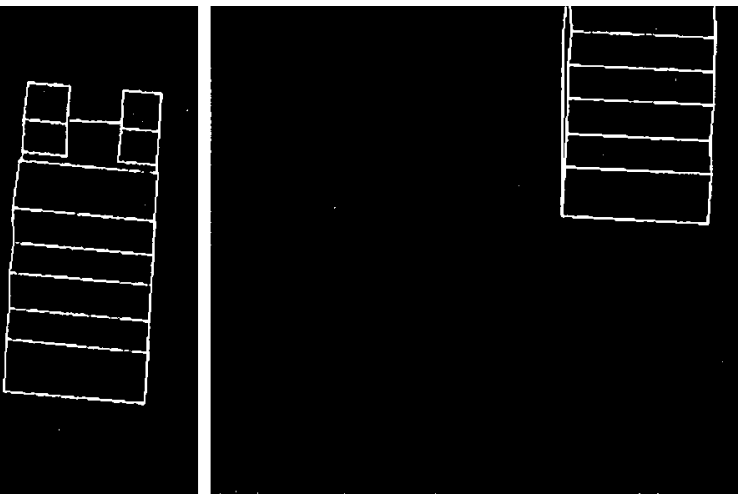

(j)

Fig. 7. (a) img1 acquired by ss1, (b) img2 acquired by ss2, (c) img3 acquired by ss3, (d) img4 acquired by ss4, (c) img5 acquired by ss5, (f) edge map of img1, (g) edge map of img2, (h) edge map of img3, (i) edge map of img4, (j) edge map of img5. 


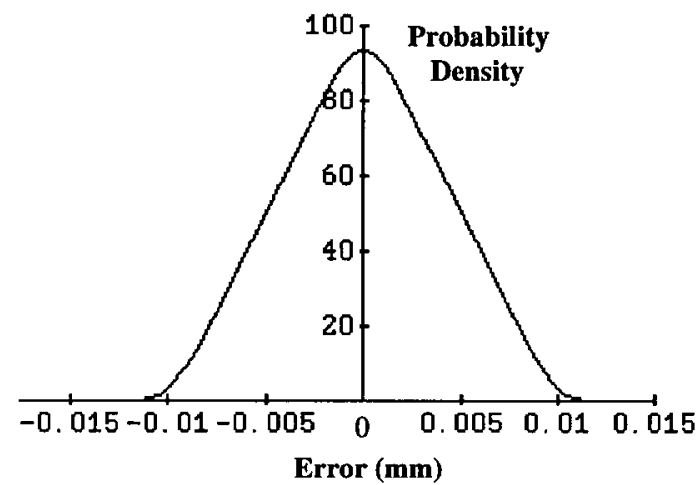

(a)

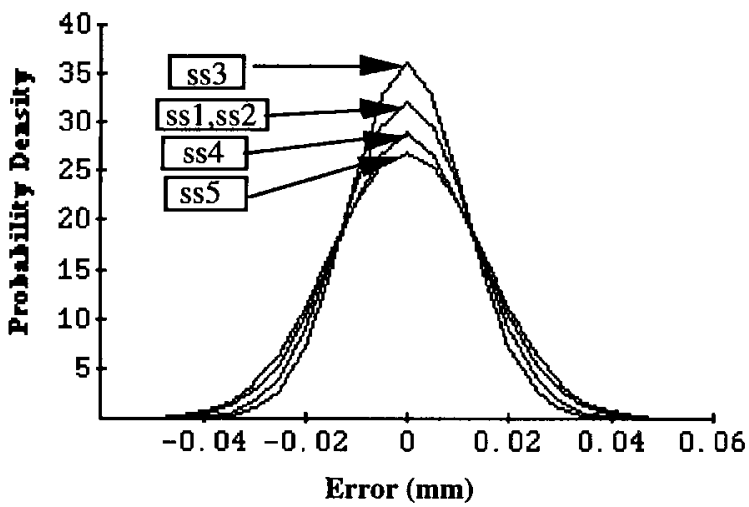

(c)

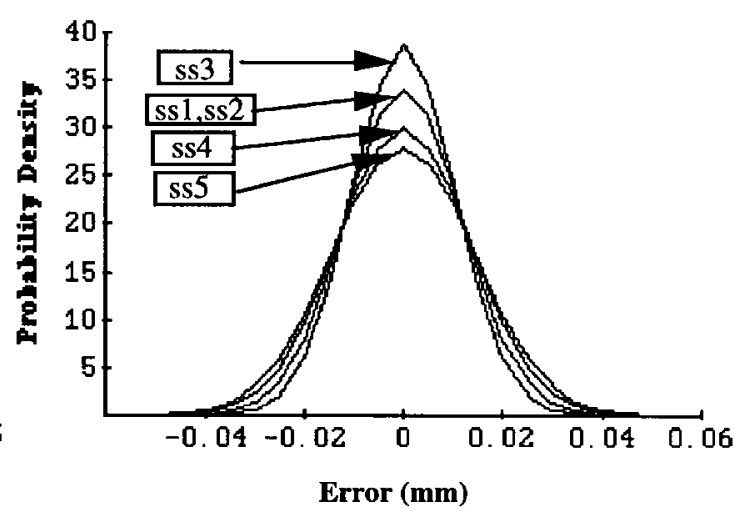

(b)

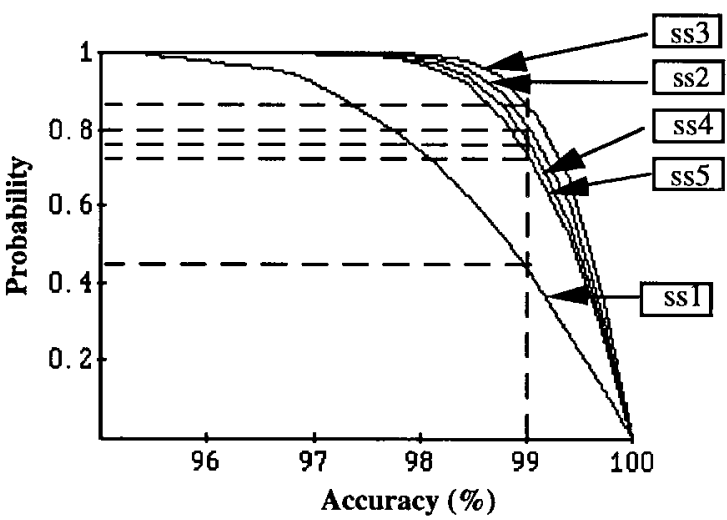

(d)

Fig. 8. (a) The pdf of the quantization error for img1, img2, img3, img4 and img5, (b) the pdf of displacement error for ss1, ss2, ss3, ss4, and ss5, (c) the pdf of the combined error of $\mathrm{ss} 1, \mathrm{ss} 2, \mathrm{ss} 3, \mathrm{ss} 4$, and ss5, (d) the accuracy of dimension by $\mathrm{ss} 1, \mathrm{ss} 2, \mathrm{ss} 3, \mathrm{ss} 4$, and $\mathrm{ss} 5$.

\section{EXPERIMENTAL RESULTS}

This section describes an experiment to investigate the effect of the choice of sensor setting (sensor orientation and location) on the dimensional inspection accuracy. Given a set of part dimensions, $M$, to be inspected, a desirable sensor setting, ss, in active vision inspection will maximize the cardinality of the subset of $M$ which would be observable and can be dimensioned from it. It also provides an acceptably high level of dimensioning accuracy for each of the elements in this subset. In dealing with different sensor settings, although some sensor settings may provide observability of a maximal set of dimensions, the (expected) dimensioning accuracy for some dimensions (edge segments) may not be acceptably high. In this experiment, the dimension $E 2$ of the part shown in Fig. 6(a) (length of the step) is dimensioned from five different sensor settings. The accuracy of the dimensional inspections are analyzed and compared. This analysis clarifies the effect of sensor settings on the dimensioning accuracy, and active vision inspection planning.

Fig. 7(a)-(e) shows five of the images (img1, img2, img3, img4, and img5) acquired from the sensor settings 1, 2, 3, 4, and 5 (ss1, ss2, ss3, ss4, and ss5). In each of ss1 and ss2, the distance between the desired entity (E2) and the sensor is approximately the same, the relative orientation between the entity and the sensor is also about the same (along the optical axes of the sensors), however, the orientation of the object relative to its center is different, causing the entity to appear differently in the image, (the edge segment is shorter on img1). For ss2 and ss3, the distance between the sensor and the desired entity in ss 2 is shorter than in ss 3 , while ss 3 has an orthogonal direction of view ( $E 2$ is perpendicular to the optical axis of ss3). The length of the desired entity in images from ss 2 and ss 3 is approximately the same. For ss3, ss4, and ss5, the distance between $E 2$ and the sensors is approximately the same. However, the object is along the optical axis of ss 3 , the object is farther away from the optical axis of ss 4 in the horizontal direction of the sensor coordinate system, and the object is farther away from the optical axis of ss5 in both horizontal and vertical directions of the sensor coordinate system. The relative orientation between $E 2$ and the sensors, ss 3 , ss 4 , and ss 5 , are significantly different. The length of $E 2$ in these images is approximately the same.

Fig. 8(a) shows the pdf of the quantization error. Since the quantization error is dependent only on the sensor resolution and the orientation of the projected segment in the image, the distributions of the quantization error for $\mathrm{ss} 1, \mathrm{ss} 2, \mathrm{ss} 3$, ss 4 , and ss5 are the same. The pdf's of errors due to displacement for ss1, ss2, ss3, ss4, and ss5 are shown in Fig. 8(b). The dimensional errors due to displacement are dependent on the relationship of the segment with respect to the image plane and its coordinate system. Because the relative orientation between $E 2$ and the sensor coordinate systems of ss 1 and ss2 is approximately the same, the distribution of the errors due to displacement for these sensor settings is approximately 
the same as shown in Fig. 8(b). In ss3, $E 2$ is farther from the sensor along the optical axis. The distribution of errors due to displacement for ss3 is narrower and its peak is higher as shown in Fig. 8(b). In ss 4 and ss5, E2 is farther away from the optical axes of the sensors, the distributions of errors due to displacement for ss4 and ss5 are wider and their peaks are lower. Combining the errors due to quantization and displacement, the pdf of total error for ss 1 , ss2, ss3, ss4, and ss5 are shown in Fig. 8(c). Using the pdf's in Fig. 8(c), we can compute the expected accuracy for dimensional inspection from ss1, ss2, and ss3 using (19). The result of this computation is plotted in Fig. 8(d).

Although the error pdf for ss1 and ss2 appear the same, the image dimension of $E 2$ from these sensor settings is different. As a result, the dimensioning accuracy from ss1 could be lower than that from ss2. For example, if we were interested in $99 \%$ accuracy, the likelihood of achieving that from ss1 is 0.44 while the likelihood for ss2 is 0.79 . The pdf of error for ss 3 is different from the other sensor settings; the likelihood of obtaining $99 \%$ accuracy is 0.87 (higher than ss1 and ss2). Intuitively, this decrease (from ss3 to ss2) in likelihood of accuracy can be attributed to the sensor being placed closer to the dimensioned entity. As distance decreases the same displacement errors introduce larger distortions in the projected coordinates and thus larger distortions in computed dimensions. Although the quantization errors will have an opposite effect, this effect may be smaller, and in any case the amount to which they cancel each other depends on two given sensor setting's relationship. Also, the introduced distortion is more serious if the inspected entity is positioned farther away from the optical axis of the sensor, which is shown in the accuracy of ss 4 and ss5. The likelihood of obtaining 99\% accuracy for ss4 and ss5 are 0.75 and 0.72 , respectively. This experiment shows the effect of the quantization and displacement errors based on the analysis presented in Sections II and III. This analysis could be helpful in determining the acceptability of given sensor settings for active vision dimensional inspection, and/or for proposing alternative sensor settings.

\section{CONCLUSION}

Although there are many advantages in using active vision inspection, there are also inherent errors. In this paper, we have concentrated on the analysis of spatial quantization errors, the errors due to sensor displacement, and their effects on the dimensional measurements of linear segments. We have developed a general analysis for the spatial quantization error in two dimensions. The range, mean, variance, and the pdf of the error in dimensional inspection of a segment are derived in terms of the resolution. A probabilistic analysis of the dimensional inspection errors due to displacement is also developed. Finally, a method for integrating the errors due to quantization and displacement makes it possible to compute the total error in active vision dimensional inspection. We have also developed an analysis for expected accuracy in dimensional measurement from different sensor settings. Accuracy is derived in terms of the resolution, the sensor location and view direction, the focal length and the model (part) dimension of the edge segment. Using this approach, we can determine the capability for inspecting different dimensions by specific sensor settings to ensure that design specifications are satisfied. Our experimental results suggest that the probabilistic based model of uncertainty in measurements gives a close match with real results. This type of analysis should be part of the system design and inspection planning procedures, leading to more accurate inspection.

\section{REFERENCES}

[1] D. H. Ballard and C. M. Brown, Computer Vision. Englewood Cliffs, NJ: Prentice-Hall, 1982

[2] S. D. Blostein and T. S. Huang, "Error analysis in stereo determination of 3-D point positions," IEEE Trans. Pattern Anal. Machine Intell., vol. PAMI-9, Nov, 1987.

[3] S. Bryson, "Measurement and calibration of static distortion of position data from 3-D trackers," in Proc. SPIE Stereoscopic Displays and Applications III, 1992, vol. 166.

[4] J. Chen and L. M. Chao, "Positioning error analysis for robot manipulators with all rotary joints," IEEE Int. Conf. Robot. Automat., San Francisco, CA, Apr. 1986.

[5] R. T. Chin, "Survey automated visual inspection: 1981-1987," Comput. Vision, Graph., Image Processing, vol. 41, pp. 346-381, 1988.

[6] C. K. Cowan, "Automated sensor placement for vision task requirements," IEEE Trans. Pattern Anal. Machine Intell., vol. PAMI-10, 1988

[7] K. Crosby, C. C. Yang, M. M. Marefat, and F. W. Ciarallo, "Optimization of sensor placement with camera constraints," in Proc. IEEE Int. Conf. Robot. Automat., Albuquerque, NM, Apr. 20-25, 1997.

[8] D. F. James and V. D. Andries, Computer Graphics: Principles and Practices. Reading, MA: Addison-Wesley, 1990.

[9] P. M. Griffin and J. R. Villalobos, "Process capability of automated visual inspection systems," IEEE Trans. Syst., Man, Cybern., vol. 22, May/June 1992.

[10] D. I. Hayclock, "Geometric precision in noise-free digital images," IEEE Trans. Pattern Anal. Machine Intell., vol. 11, pp. 1065-1075, 1989.

[11] C. Ho, "Precision of digital vision systems," IEEE Trans. Pattern Anal. Machine Intell., vol. PAMI-5, Nov. 1983.

[12] A. Huertas and C. Medioni, "Detection of intensity changes with subpixel accuracy using Laplacian-Gaussian masks," IEEE Trans. Pattern Anal. Machine Intell., vol. PAMI-8, Sept. 1986.

[13] S. A. Hutchinson, R. L. Cronwell, and A. C. Kak, "Planning sensing strategies in robot work cell with multi-sensor capabilities," IEEE Trans. Robot. Automat., vol. 5, 1989.

[14] K. Jensen and D. Anastassiou, "Subpixel edge localization and the interpolation of still images," IEEE Trans. Image Processing, vol. 4, Mar. 1995.

[15] B. Kamgar-Parsi and B. Kamgar-Parsi, "Evaluation of quantization error in computer vision," IEEE Trans. Pattern Anal. Machine Intell., vol. 11, Sept. 1989.

[16] M. Kisworo, S. Venkatesh, and G. West, "Modeling edges at subpixel accuracy using the local energy approach," IEEE Trans. Pattern Anal. Machine Intell., vol. 16, Apr. 1994.

[17] E. Lyvers, O. R. Mitchell, M. L. Akey, and A. P. Reeves, "Subpixel measurements using a moment-based edge operator," IEEE Trans. Pattern Anal. Machine Intell., vol. 11, Dec. 1989.

[18] P. J. MacVicar-Whelan and T. O. Binford, "Line finding with subpixel precision," in Proc. SPIE Techniques Appl. Image Understanding, 1981, vol. 281.

[19] M. M. Marefat and R. L. Kashyap, "Image interpretation and object recognition in manufacturing," IEEE Contr. Syst., vol. 11, Aug. 1991.

[20] _ "Feature-based computer integrated inspection," in Proc. 1993 ASME Int. Comput. Eng. Conf., San Diego, CA, Aug. 8-12, 1993.

[21] C. H. Menq and J. H. Borm, "Statistical measure and characterization of robot errors," in Proc. IEEE Int. Conf. Robot. Automat., 1988.

[22] C. H. Menq, H. T. Yau, and G. Y. Lai, "Automated precision measurement of surface profile in CAD-directed inspection," IEEE Trans. Robot. Automat., vol. 8, Apr. 1992.

[23] C. W. Moon, "Error analysis for dimensional measurement using computer vision techniques," IEEE Instrum. Meas. Technol. Conf., San Diego, CA, 1988, pp. 371-376.

[24] H. D. Park and O. R. Mitchell, "CAD based planning and execution of inspection," in Proc. IEEE Comput. Vision Pattern Recog. Conf., Ann Arbor, MI, 1988 
[25] R. P. Paul, Differential Relationships, Robot Manipulators: Mathematics, Programming, and Control. Cambridge, MA: MIT Press, 1989, ch. 4.

[26] G. M. Radack and F. L. Merat, "The integration of inspection into the CIM environment," in Proc. Hawaii Int. Conf. Syst. Sci., 1990, pp. $445-462$.

[27] J. Renders, E. Rossignol, M. Becquet, and R. Hanus, "Kinematic calibration and geometrical parameter identification for robots," IEEE Trans. Robot. Automat., vol. 7, Dec. 1991.

[28] S. Yi, R. M. Haralick, L. Shapiro, "Automatic sensor and light source positioning for machine vision," in Proc. 10th Int. Conf. Pattern Recog., Alantic City, NJ, June 2-3, 1991.

[29] R. C. Smith and P. Chessman, "On the representation and estimation of spatial uncertainty," Int. J. Robot. Res., vol 5, no. 4, 1986.

[30] S. F. Su and C. S. G. Lee, "Manipulation and propagation of uncertainty and verification of application of actions in assembly tasks," IEEE Trans. Syst., Man, Cybern., vol. 22, Nov./Dec. 1992.

[31] W. K. Veitschegger and C. Wu, "A method for calibrating and compensating robot kinematic errors," in Proc. IEEE Int. Conf. Robot. Automat., 1987.

[32] Z. Yan and C. H. Menq, "Coordinate sampling and uncertainty analysis for computer integrated manufacturing and dimension inspection," in Proc. 1993 NSF Design Manufact. Syst. Conf., Charlotte, NC, Jan. 6-8, 1993, pp. 1705-1711.

[33] C. C. Yang, M. M. Marefat, and R. L. Kashyap, "Active visual inspection on CAD models," IEEE Int. Conf. Robot. Automat., San Diego, CA, May 8-13, 1994.

[34] _ "Analysis of errors in dimensional inspection based on active vision," in Proc. SPIE Int. Symp. Intell. Robot Comput. Vision XIII: 3-D Vision, Product Inspection, Active Vision, Cambridge, MA, Oct. 31-Nov. 4, 1994.

[35] K. Tarabanis, R. Y. Tsai, and P. K. Allen, "Analytic characterization of the feature detectability constraints of resolution, focus, and field-ofview for vision sensor planning," CVGIP: Image Understanding, vol. 59, no. 3, pp. 3400-358, May 1994.

[36] - "The MVP sensor planning system for robotic vision tasks," IEEE Trans. Robot. Automat., vol. 11, Feb. 1995.

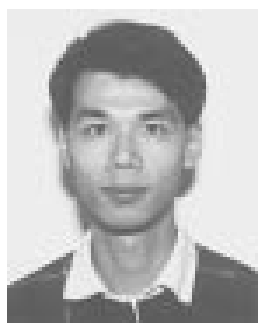

Christopher C. Yang (S'90-M'96) was born in Hong Kong. He received the B.S., M.S., and Ph.D. degrees in electrical engineering from the University of Arizona, Tucson, in 1990, 1992, and 1997, respectively.

$\mathrm{He}$ is an Assistant Professor in the Department of Computer Sciences, University of Hong Kong. From 1992 to 1997, he was a Research Associate in the Intelligent Systems Laboratory, Department of Electrical and Computer Engineering, University of Arizona. From 1995 to 1997, he was a Research Scientist in the Artificial Intelligence Laboratory, Department of Management Information Systems, University of Arizona. From 1991 to 1992, he worked in Multidimensional Image Processing Laboratory, Deparment of Electrical and Computer Engineering, University of Arizona, as a Research Assistant. His current research interests are computer integrated manufacturing and inspection, constraint network, visualization, color image processing, digital library, and internet agent.

Dr. Yang is a member of the Program Committee for the 1997 IEEE International Conference on Systems, Man, and Cybernetics, and the Organizing Committee for the 1998 Third Asian Conference on Computer Vision.

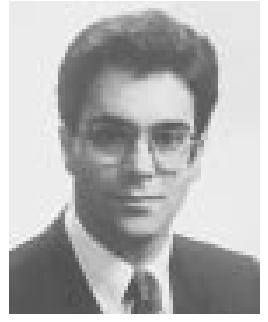

Michael M. Marefat (S'89-M'92-SM'98) received the B.A. degree in mathematical sciences from Rice Universtiy, Houston, TX, in 1986, and the M.S. and $\mathrm{Ph} . \mathrm{D}$. degrees in electrical engineering from Purdue University, West Lafayette, IN, in 1988 and 1991, respectively.

He is the Director of the Intelligent Systems Laboratory and a member of faculty in the Departmen of Electrical and Computer Engineering, University of Arizona, Tucson. He has been involved in research in the areas of knowledge-based systems, visualization, computer graphics, machine vision, and computer-aided design for the last ten years. He is the author or co-author of about 100 refereed research articles. His research has been supported by the National Science Foundation by NASA, and by the state of Arizona. He has been Supervisor of more than 12 Master theses and Ph.D. dissertations. His work previous experience includes the Schlumberger Laboratory for Computer Science, the Purdue Engineering Research Center for Intelligent Manufacturing Systems, and the Baylor Digital Imaging Laboratory.

Dr. Marefat is a member of AAAI.

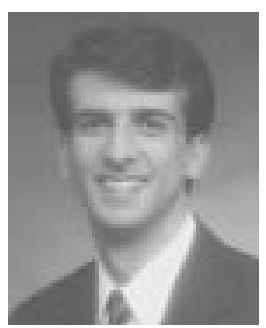

Frank W. Ciarallo (S'86-M'91) received the B.S. degree in electrical engineering and engineering and public policy, and the M.S. and Ph.D. degrees in manufacturing and operation systems, all from Carnegie Mellon University, Pittsburgh, PA, in 1986, 1988, and 1993, respectively.

$\mathrm{He}$ is an Assistant Professor in the Department of Systems and Industrial Engineering, University of Arizona, Tucson. His research interests include production planning and inventory control with process uncertainties, optimization and uncertainties in inspection planning, and applications of simulation

Dr. Ciarallo is a member of the Institute for Operations Research and Management Sciences. 